\title{
Construction of the Elements of an Arm for Mobile Handling by CNC machining
}

\section{Construcción de Elementos de un Brazo para Manipulación Móvil mediante mecanizado CNC}

\section{Angel Silva¹, Víctor Andaluz², Angel Guamán ${ }^{1}$, Miguel Pérez¹, and Paúl Romero ${ }^{3}$}

${ }^{1}$ ESPOCH, Proyecto CEDIA, Facultad de Mecánica, Riobamba, Ecuador ${ }^{2} E S P E$, Proyecto CEDIA, Departamento de Energía y Electrónica, Quito, Ecuador ${ }^{3}$ ESPOCH, Proyecto CEDIA, Facultad de Informática y Electrónica, Riobamba, Ecuador

\section{Abstract}

Corresponding Author:

Angel Silva

angelsilvaec@hotmail.com

Received: 4 December 2018

Accepted: 5 December 2018

Published: 27 December 2018

Publishing services provided by Knowledge $\mathrm{E}$

(c) Angel Silva et al. This article is distributed under the terms of the Creative Commons

Attribution License, which permits unrestricted use and redistribution provided that the original author and source are credited.

Selection and Peer-review under the responsibility of the SIIPRIN-CITEGC Conference Committee.
The control of a manipulator arm requires that the designed elements be constructed with excellent precision which can be achieved with the use of CNC machines. This article presents the required machining techniques and the procedure used in the construction of each of the parts of the system, which has specific characteristics according to its future application.

\section{Resumen}

El control de un brazo manipulador requiere que los elementos diseñados sean construidos con excelente precisión la cual puede ser lograda con el uso de las máquinas CNC. En este trabajo se presenta las técnicas de mecanizado requeridas y el procedimiento usado en la construcción de cada una de las partes del sistema, el cual posee características específicas según su futura aplicación.

Keywords: CNC, CAM, milling machine, Interpolation, G Code, machining Palabras clave: CNC, CAM, fresadora, Interpolación, Código G, mecanizado

\section{G OPEN ACCESS}

\section{Introducción}

La Tele Operación Bilateral Cooperativa de múltiples manipuladores móviles, requiere de sistemas autónomos consistentes en un robot manipulador (o brazo robótico) ubicado sobre una plataforma móvil (con ruedas), los cuales desempeñan tareas específicas. 
Dentro de éste proyecto, cooperativo entre varias instituciones, las piezas requeridas para el ensamble del brazo manipulador deben tener un proceso de fabricación de calidad logrando con ello una precisión en sus funciones y movimientos.

El presente trabajo, se centra en el mecanizado de algunos elementos requeridos para el ensamble de un brazo manipulador para que cumpla con determinadas características predefinidas (de diseño y resistencia).

El proceso de mecanizado, que puede ser logrado con una máquina CNC, tiene esas características de fiabilidad, precisión y rapidez. Se usará para el efecto la fresadora BRIDGEPORT VMC 800 ubicada en el centro de mecanizado de la Facultad de Me-cánica de Escuela Superior Politécnica de Chimborazo, institución, que entre otras similares, forma parte del proyecto cooperativo.

\section{Materiales y métodos}

\subsection{Mecanizado en máquinas CNC}

Una máquina de Control Numérico por Computadora o CNC es un sistema que permi-te controlar la posición, la forma de desplazamiento, la velocidad y otros parámetros de la herramienta montada en ella, en cualquier instante, lo cual permite mecanizar piezas que poseen forma compleja y que requieren gran precisión. Todo estas caracte-rísticas del CNC, requieren de un software profesional CAM (Computer-Aided Manu-facturing / Fabricación asistida por computadora), el cual permite la generación auto-mática de programas de CNC, para realizar el mecanizado de las piezas requeridas.

Para la realización de los programas de maquinados se tiene dos métodos [1]:

\subsubsection{Manual}

En este caso la programación la realiza la persona encargada de esta tarea, utilizando sus habilidades de programación, y solo se usa cuando se necesita mecanizar piezas de poca complejidad.

\subsubsection{Automática}

aquí la programación se obtiene por medio del software CAM que realiza los cálculos y genera la programación a partir de un archivo CAD de la pieza a mecanizar, siendo su ventaja el realizar programaciones para mecanizar piezas con geometrías complejas. 


\subsection{Fresadora CNC}

Son muy similares a las convencionales y poseen las mismas partes móviles, es decir, la mesa, el cabezal de corte, el husillo y los carros de desplazamiento lateral y transversal. Sin embargo, no presentan palancas ni manivelas para accionar estas partes móviles, sino una pantalla insertada en un panel repleto de controles y una caja metálica donde se alojan los componentes eléctricos y electrónicos que regulan el funcionamiento de motores destinados a efectuar el mismo trabajo que hacían las palancas y manivelas de las viejas máquinas. Entre estos componentes se encuentra el CNC, que es una computadora principalmente responsable de los movimientos de la fresadora a través del correspondiente software. La combinación de electrónica y motores o servomotores de accionamiento es capaz de lograr todas las operaciones de fresado posibles [2] y [3].

\subsection{Estándares de Instrucciones}

Estos códigos permiten programar las funciones de movimiento de la máquina herramienta para llevar a cabo las operaciones de mecanizado; entre estos tenemos:

$\mathrm{N}$ : Número de bloque.

G: Función preparatoria.

$\mathrm{X}$ : Coordenada en $\mathrm{X}$.

Y: Coordenada en Y.

Z: Coordenada en Z.

T: No. de herramienta.

I: Localización en X del centro del arco.

J: Localización en Y del centro del arco.

K: Localización en Z del centro del arco.

S: Velocidad del husillo.

F: Velocidad de avance de corte.

M: Función Miscelánea. 


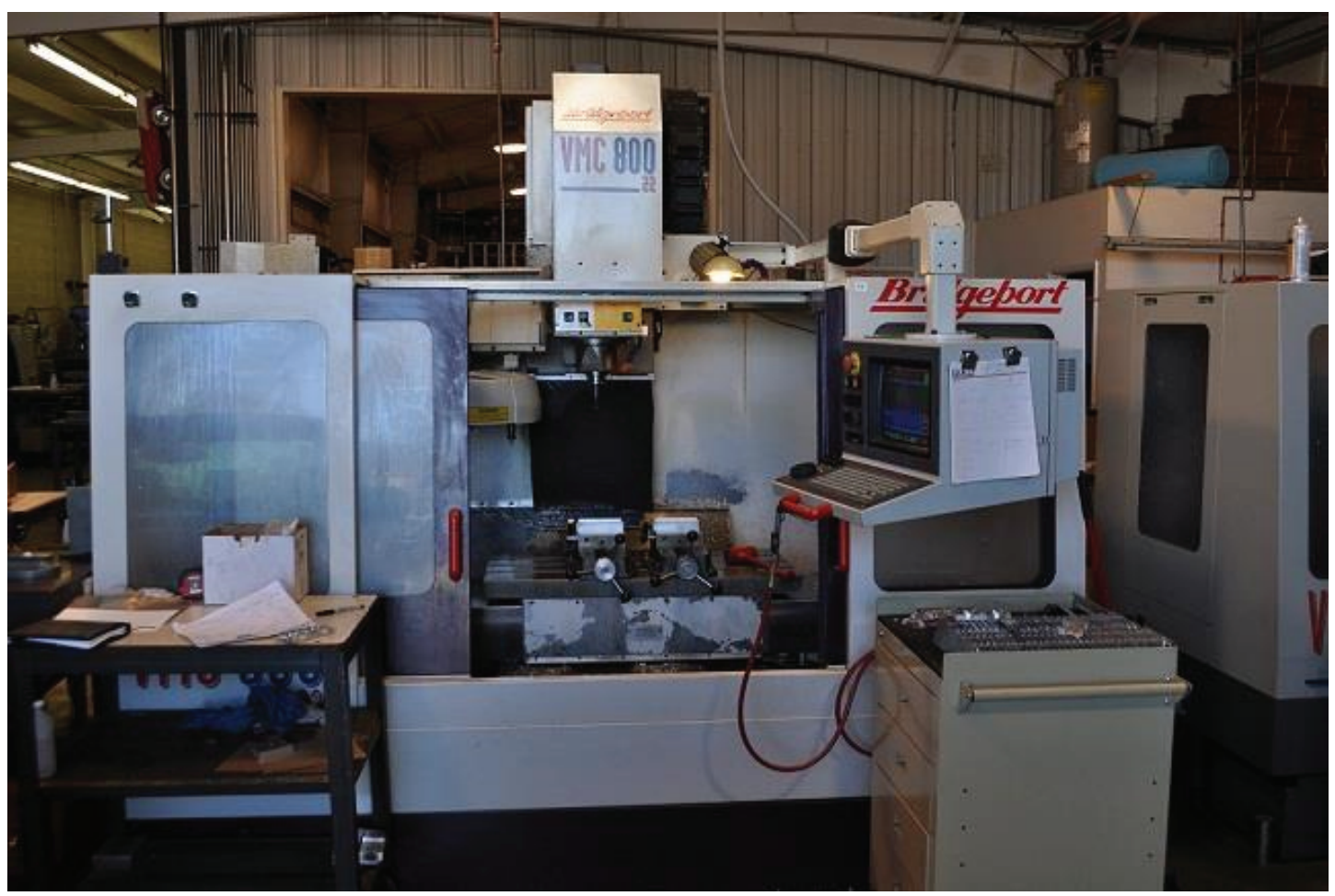

FIGURA 1: Centro de mecanizado vertical de tres ejes BRIDGEPORT VMC 800.

Los códigos G o Generales son usados para especificar el tipo de movimiento u opera-ción a ejecutarse; los principales códigos $\mathrm{G}$ requeridos son:

Goo: Posicionamiento rápido.

Go1: Interpolación lineal.

Go2: Interpolación circular (Sentido horario).

Go3: Interpolación circular (Sentido antihorario).

G17: Selección del plano XY.

G15: Cambio a coordenadas polares.

G20: Sistema Imperial.

G21: Sistema métrico.

G54: Decalaje de origen.

G81: Taladrado.

G94: Velocidad de corte expresada en avance por minuto.

G95: Velocidad de corte expresada en avance por revolución. 
Los códigos M o Misceláneos designan funciones misceláneas o secundarias que con-trolan el funcionamiento del husillo de la maquina CNC.

Moo: Paro del programa.

Mo3: Giro del husillo en sentido horario.

Mo4: Giro del husillo en sentido anti horario.

Mo6: Cambio programado de herramienta

Mo8: Activación del refrigerante

Mo9: Desactivación del refrigerante.

M30: Fin del programa y regreso al inicio del mismo.

A continuación, se muestra la estructura de un bloque de programación CNC:

$\begin{array}{llllllllll}N & G & X & Y & Z & F & S & T & M\end{array}$

Dentro de cada bloque debe mantenerse este orden. Sin embargo, no es necesario que estén presentes todos los ítems.

\subsection{Técnicas de Fresado}

El fresado se realiza con herramientas de corte rotatorio que poseen múltiples filos las cuales ejecutan movimientos de avance programados contra una pieza en prácticamente cualquier dirección [5]. Las principales operaciones a realizarse son:

- Fresado en escuadra.

- Planeado

- Fresado de perfiles

- Taladrado (Agujeros y cavidades)

- Achaflanado.

A continuación, se definen algunos de estos conceptos.

\subsubsection{Planeado}

Es la operación más sencilla de fresado, en la que la pieza en bruto se mueve recto hacia la fresa, de tal manera que el plano en que esta avanza y el eje de rotación sean perpendiculares, dejando una superficie plana sobre el plano en que la fresa corta. 


\subsubsection{Fresado}

El fresado es la operación de mecanizado que más se utiliza en una Fresadora mediante una herramienta cilíndrica parecida a una broca (denominada fresa), pero generalmente sin punta cónica. Esta herramienta elimina material por la superficie cilíndrica, y es ideal para realizar mecanizados en superficies planas y para realizar un agujero de casi cualquier forma en una pieza de partida.

\subsubsection{Taladrado}

En una operación de taladrado, la broca se desplaza a un punto específico en la pieza y se taladra a profundidades específicas. Se debe instalar el taladro apropia-do para los distintos tamaños de agujero.

\subsection{Selección, características y propiedades del material para la fabricación}

En función de los requerimientos del diseño y del mecanizado, se requiere de un material que cumpla con las siguientes características mecánicas [6]:

Módulo de elasticidad $>7000 \mathrm{~N} / \mathrm{mm}^{2}$

Resistencia a la tracción $>500 \mathrm{~N} / \mathrm{mm}^{2}$

En función del cumplimiento de estas características y otros parámetros como la disponibilidad del material y la tecnología de fabricación, se escoge el material Duraluminio para su manufactura.

El Duraluminio es una aleación de aluminio con variados porcentajes de cobre, magne-sio, silicio y pequeñas trazas de hierro. Se caracteriza por tener excelentes propiedades mecánicas después de algunos días de reposo una vez templado a unos $500^{\circ} \mathrm{C}$. Tienen una resistencia mecánica muy elevada a temperatura ambiente, sin embargo, su resistencia a la corrosión, soldabilidad y aptitud para el anodizado son bajas. Se emplean en la industria aeronáutica y de automoción entre otras, por su gran ligereza unida a elevada resistencia, además poseen poca resistencia a la abrasión (fácil mecanizado) [7].

Su composición típica es: cobre (0.45\%-1.5\%), magnesio (0.45\%-1.5\%), manganeso (0.6\%-0.8\%) y silicio (0.5\%-0.8\%) [8].

En la tabla 1 y 2, se muestran las propiedades físicas y mecánicas, respectivamente, del material seleccionado: 
TABLA 1: Propiedades físicas del duraluminio PRODAX.

\begin{tabular}{|c|c|}
\hline Propiedad & Valor \\
\hline Densidad $\mathrm{kg} / \mathrm{m}^{3}$ & 2830 \\
\hline Módulo de eleasticidad $\mathrm{N} / \mathrm{mm}^{2}$ & 71500 \\
\hline Coeficiente de expansion térmica po ${ }^{\circ} \mathrm{C}$ de $20^{\circ} \mathrm{C}$ a $100^{\circ} \mathrm{C}$ & $23 \times 10^{-6}$ \\
\hline Conductibilidad térmica $\mathrm{W} / \mathrm{m}{ }^{\circ} \mathrm{C}$ & 165 \\
\hline Calor específico $/ \mathrm{kg}^{\circ} \mathrm{C}$ & 890
\end{tabular}

TABLA 2: Propiedades mecánicas del duraluminio PRODAX en placa.

Placa (espesor $\mathrm{mm}$ ) Resistencia a la tracción $\mathrm{N} / \mathrm{mm}^{2}$ Límite de Fluencia N/mm² $>10-50$ 590 550

\subsection{Cálculos de datos de corte}

El fresado se realiza con herramientas de corte rotatorio que poseen múltiples filos las cuales ejecutan movimientos de avance programados contra una pieza en prácticamen-te cualquier dirección. A continuación, se presentan dos características principales del mecanizado, como son la velocidad del husillo y la velocidad de mesa o velocidad de avance:

- Velocidad del husillo:

$$
n=\frac{v_{c} \times 100}{\pi \times D_{c}}
$$

Donde vc es la velocidad de corte en $\mathrm{m} / \mathrm{min}$, Dc es el diámetro de la fresa en $\mathrm{mm}$ y $n$ es la velocidad del husillo en RPM [4] y [5].

- Velocidad de mesa o velocidad de avance:

$$
V f=n \times F z \times z n
$$

Donde $\mathrm{Vf}$ es la velocidad de avance de la mesa en $\mathrm{mm} / \mathrm{min}, \mathrm{fz}$ es el avance por diente en mm, $n$ es la velocidad del husillo en RPM y zn es el número de dientes de la fresa [4] y [5].

\section{Resultados y discusión}

\subsection{Proceso de mecanizado de las piezas}

Las piezas que forman parte del brazo fueron previamente diseñadas y modeladas en un software $C A D$, del cual se partió para poder obtener los códigos de programación 
por medio del software Mastercam V para luego realizar la respectiva validación de la simulación del movimiento de la herramienta en el editor CIMCO Edit V7.

Para cada pieza se obtuvola programación respectiva de la maquina CNC con los respectivos parámetros de esta, cada uno de los archivos CAD fueron procesados en el programa CAM y se realizó la simulación de cada una de las operaciones que se requería en cada una de las piezas, para detectar posibles errores en el proceso de mecanizado.

Ejemplo de la programación del CNC en la cual se realiza la operación de faceteado para el material en bruto.

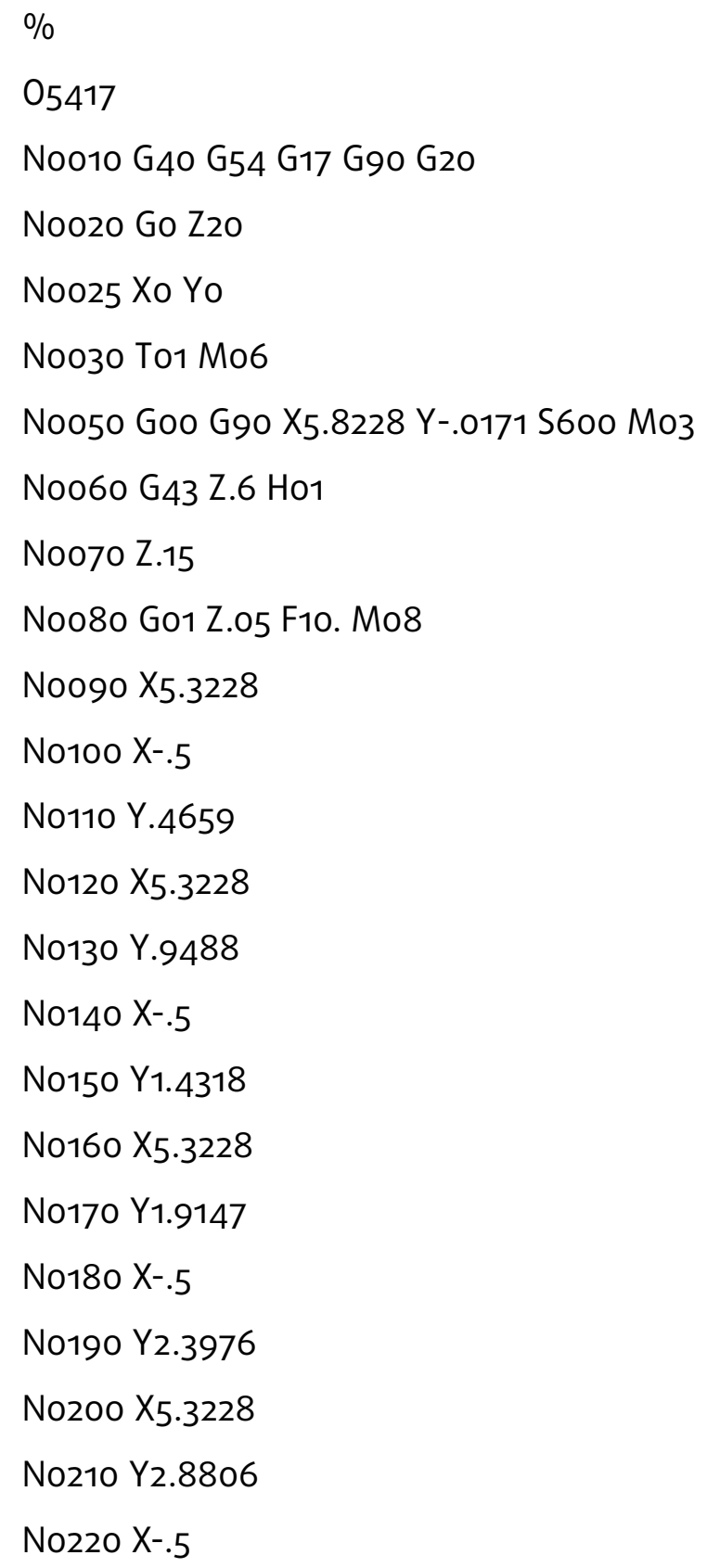


No230 Y3.3635

No240 X5.3228

No250 $\times 5.8228$

No260 Z.15

No270 Goo Z.6

N2910 Goo Z.6

$\mathrm{N} 2920 \mathrm{Mo2}$

$\%$

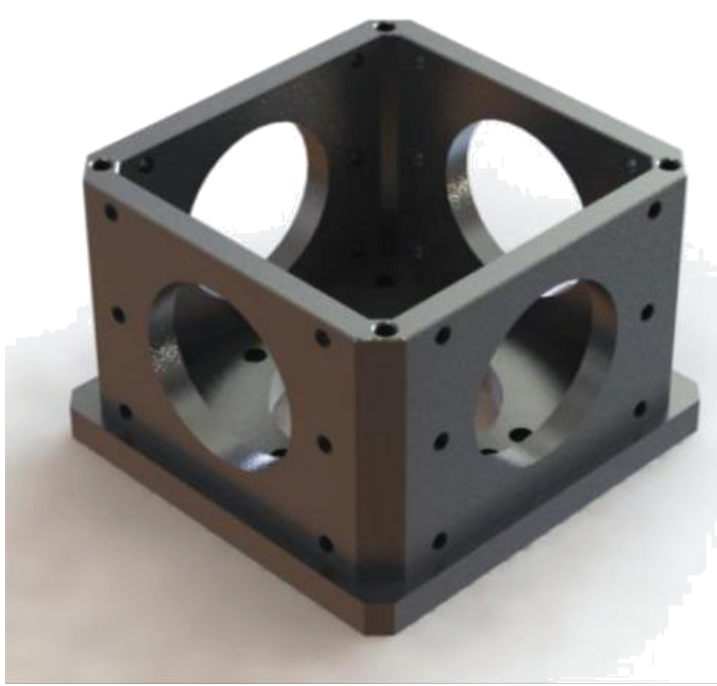

(a)

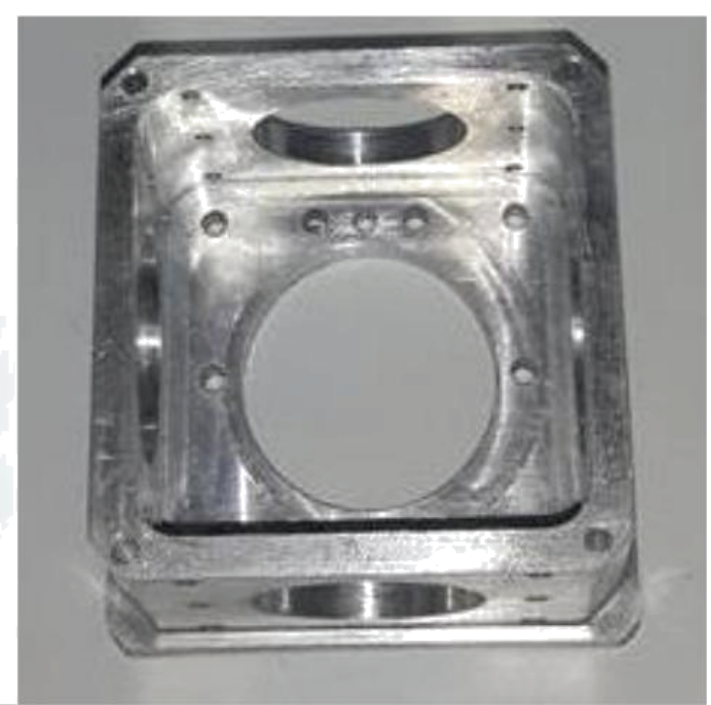

(b)

FIGURA 2: Pieza base 1; (a) Pieza modelada en el software SolidWorks; (b) Pieza mecanizada en la maquina CNC.

El material inicialmente se facetea, operación necesaria para obtener paralelismo entre sus caras, debido a la forma de las piezas, posteriormente se hace una operación de vaciado de las cavidades y luego el contorneado de la pieza, estas dos operaciones varían según el elemento a fabricar, luego se libera la pieza y se realiza el punteado guía del centro de los agujeros para luego proceder a las operaciones de taladrado y roscado de estos.

El procedimiento fue el mismo para todas las piezas, la ventaja de la maquina CNC es de brindar excelentes acabados superficiales, además de la precisión con la que realiza el proceso; posterior a esta operación, se realiza la verificación de las dimensiones. 


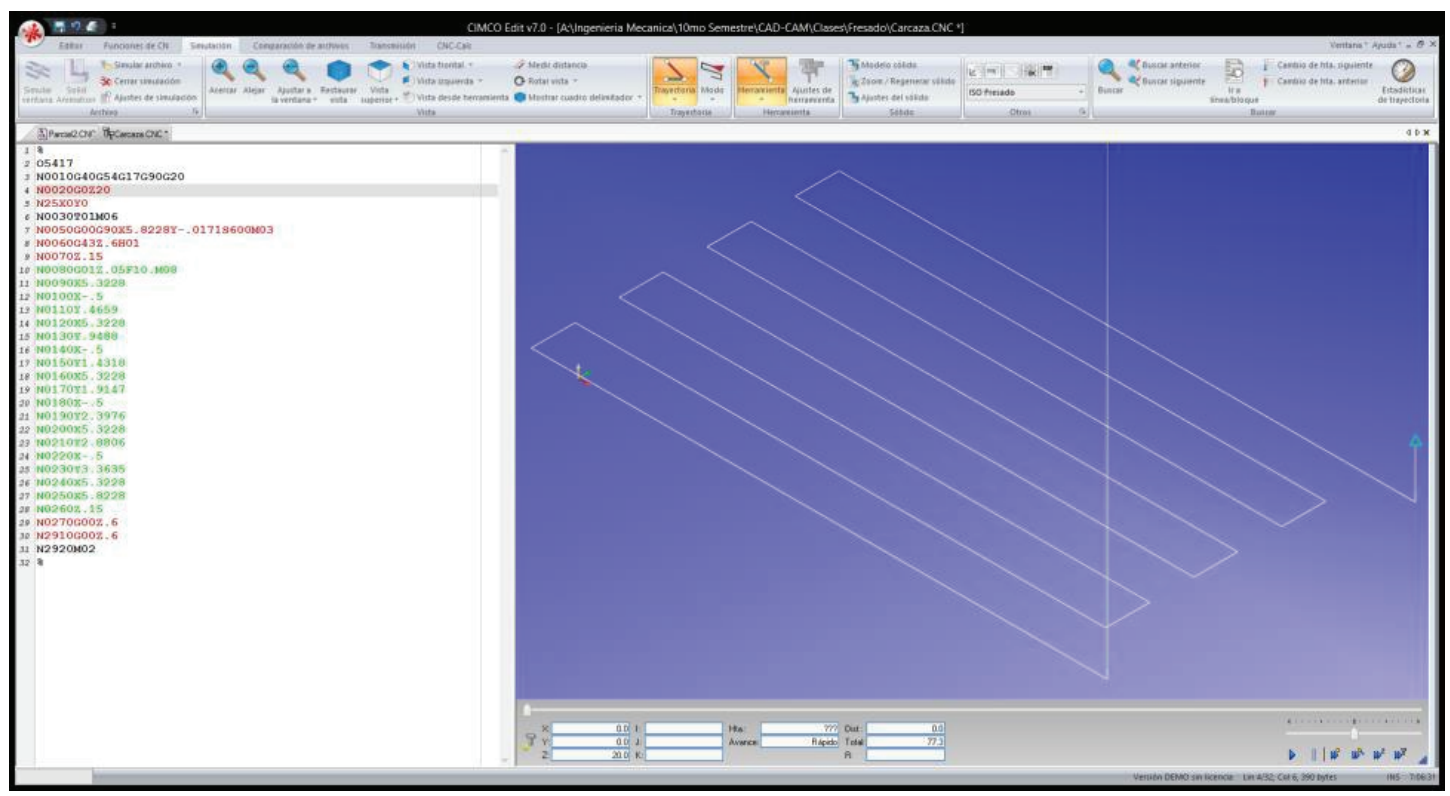

FIGURA 3: Simulación de la programación CNC para la operación de Faceteado en el Software CIMCO Edit V7.

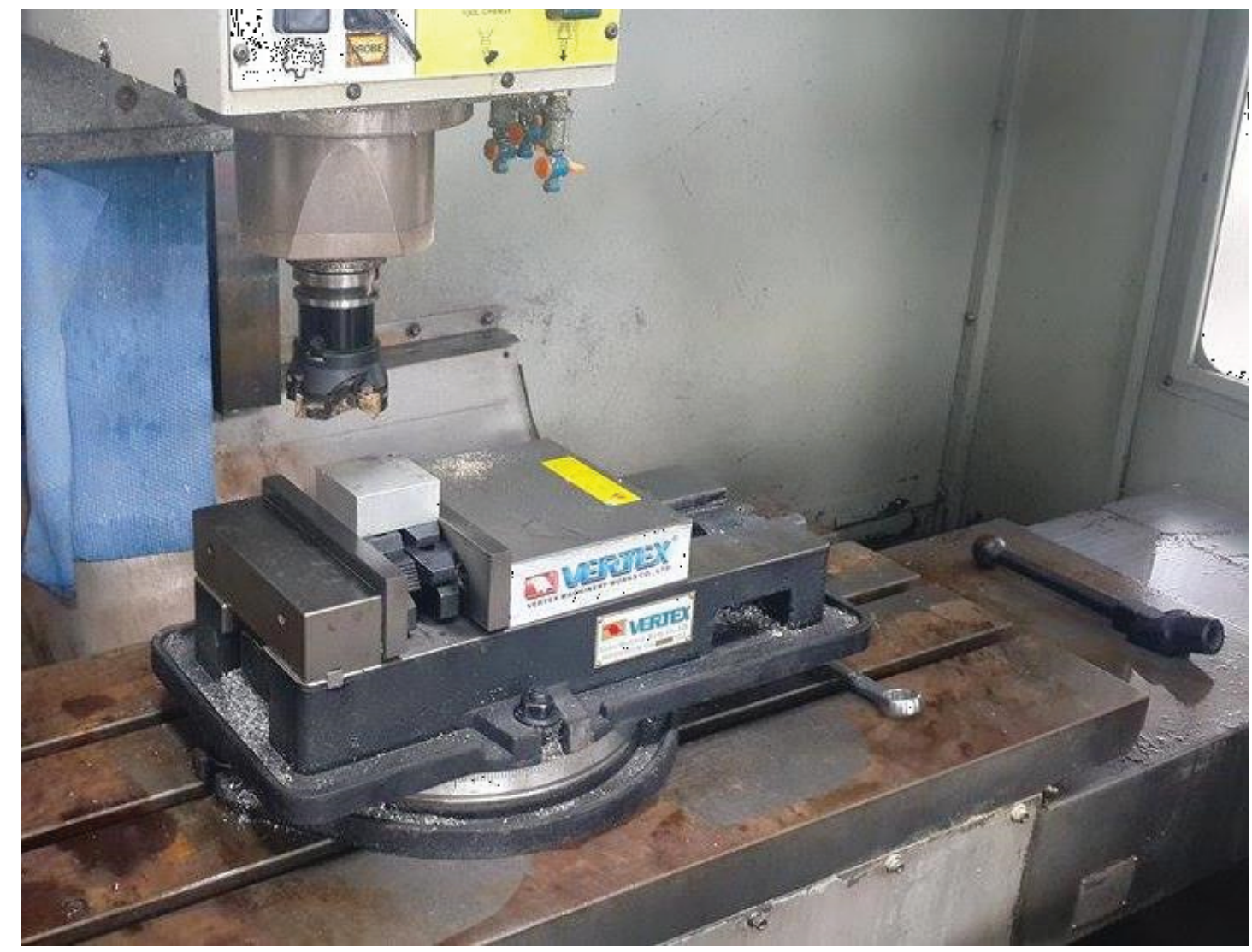

FıguRA 4: Operación de faceteado de las piezas en bruto para obtener superficies planas en ésta. 


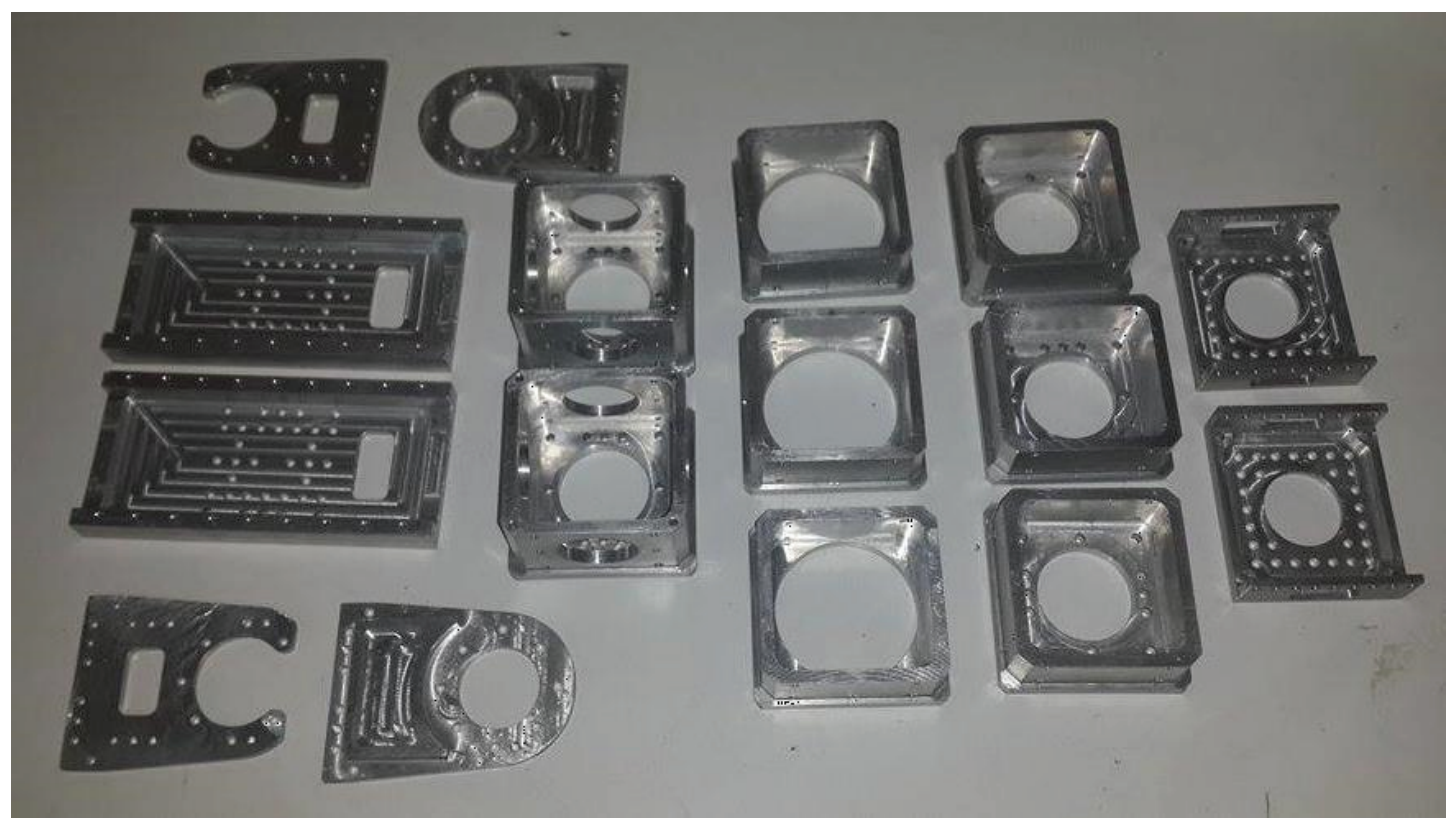

Figura 5: Fresado de las cavidades y contornos de la pieza en el centro de mecanizado vertical de tres ejes.

\section{Conclusiones}

- El proceso de mecanizado de las piezas requeridas para el brazo del manipulador móvil fue llevado a cabo de manera minuciosa para lograr excelentes acabados finales y que las características y propiedades del material no se vea afectado en el proceso de corte lo que da seguridad de la calidad de la pieza.

- Con la comprobación de calidad en la fabricación, las pruebas en el simulador CAD indican que no existe interferencia en los movimientos, permitiendo un correcto funcionamiento de los manipuladores móviles y la cooperación entre estos.

- El procedimiento descrito indica que se puede utilizar la tecnología CNC para una óptima fabricación de sistemas robóticos, en función de los requerimientos previos.

- Se demostró, además, que el material elegido puede ser fácilmente mecanizado para la fabricación de prototipos mecánicos sin afectar a sus propiedades mecánicas y a la ingeniería del proyecto.

- Se requieren, además, por parte del operador del CNC, conocimientos y experiencia en el manejo de la maquinaria para evitar posibles fallas, accidentes y afectaciones que incidan en el buen desarrollo del proyecto. 


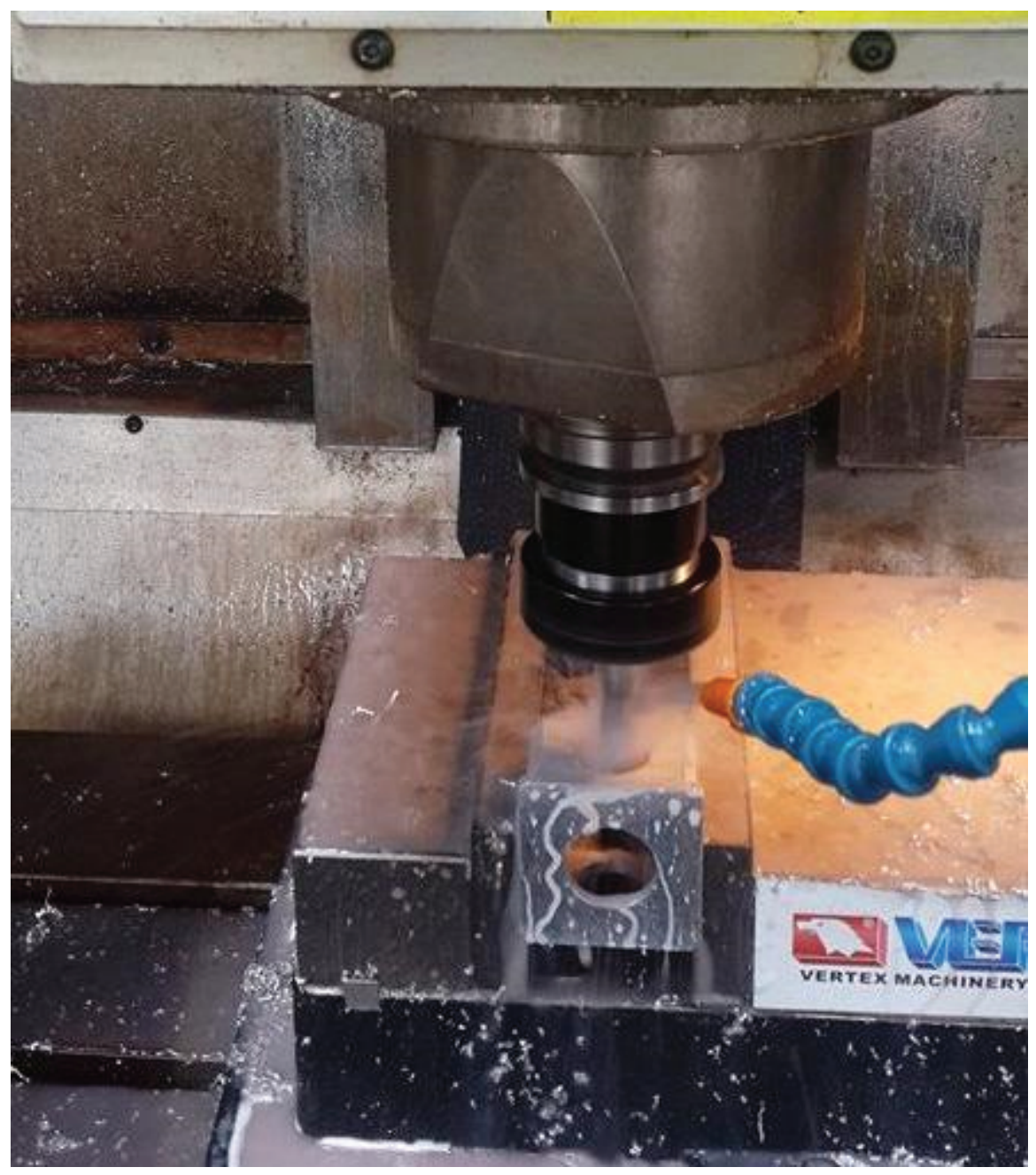

Figura 6: Piezas del brazo del manipulador móvil.

\section{Recomendaciones y perspectivas de la investigación}

- Se requiere, por parte del operador del CNC, conocimientos y experiencia en el manejo de la maquinaria para evitar posibles fallas, accidentes y afectaciones que incidan en el buen desarrollo del proyecto.

- Se recomienda el uso de sistemas de seguridad en la programación del CNC para evitar posibles contratiempos en el mecanizado.

- Se requiere la disponibilidad de herramientas para el mecanizado para lograr tiempos óptimos de fabricación.

- Se recomienda el uso de materiales alternativos, cómo el plástico y la fibra, para mejorar el diseño y la posterior construcción de los elementos. 


\section{Referencias}

[1] J. J. Peters, "Introducción al control numérico computarizado (CNC)", Creative Commons, Dic 2013.

[2] Bruce, J. Cruz, J. A. Rico, U. Scharer. Ingeniería de Manufactura 2008. México: Compañía Editorial Continental.

[3] M. Cruz, Control Numérico por Computadora (CNC). Queretaro: Instituto Tecnológico de Queretaro, 2014.

[4] N. Larburu, "Máquinas prontuario. Técnicas, máquinas, herramientas", Parainfo, 1997.

[5] S. Kalpakjian, S. R. Schmid, "Manufactura Ingeniería y Tecnología", Prentice Hall, 2002.

[6] R. Robayo, D. Pazmiño, "Diseño y construcción de un sistema mecánico para un robot ma-nipulador móvil en el proyecto de investigación "Tele-operación bilateral cooperativo de múltiples manipuladores móviles, aprobado por el Consorcio ecuatoriano para el Desarrollo de Internet Avanzado - CEDIA".

[7] http://www.ivanbohman.com.ec/wp-content/uploads/2016/o2/prodax.pdf.

[8] http://www.fullmecanica.com/definiciones/d/350-duraluminio. 